\title{
Combining Ability, Gene Action and Heterotic Expression Studies for Yield Contributing Traits in Tomato
}

\author{
K. V. Manjunath* and Subhamoy Sikder \\ Department of Vegetable and Spice Crops, Uttar Banga Krishi Viswavidyalaya Pundibari, \\ Coochbehar, West Bengal, India \\ *Corresponding author
}

\begin{abstract}
A B S T R A C T
Investigation was carried out at the experimental field of Department of Vegetable and Spice Crops, Uttar Banga Krishi Viswavidyalaya, Pundibari, Coochbehar, West Bengal with seven lines, four testers and twenty-eight cross combinations derived from line $\times$ tester model to study the genetic performance of tomato genotypes. The significant variances due to lines and testers, line $v s$. tester and parent $v s$. hybrid indicated the importance of their contribution towards general combining ability (GCA), specific combining ability (SCA) and presence of heterotic expression for important characters respectively. The magnitude of predictability ratio for all the traits studied was very less than unity indicated preponderance of non-additive gene action for the inheritance of all the traits. Among lines Arka Alok, Arka Saurabh, Arka Vikas and Arka Meghali and among testers Berika and Alisa Craig possess good GCA. Arka Saurabh $\times$ Alisa Craig, Arka Ashish $\times$ Berika and Arka Vikas $\times$ Berika crosses identified as best with good SCA. Arka Saurabh $\times$ Alisa Craig followed by Arka Alok $\times$ Berika, Arka Meghali $\times$ Berika and Arka Ahuti $\times$ Berika were best identified crosses with heterotic expression for the yield contributing traits which was due to the involvement of the parents having high GCA and crosses with good specific combining ability.
\end{abstract}

Keywords

Analysis of

Variance, Genetic

variance, GCA,

Heterosis, Line $\times$

Tester, SCA,

Tomato

Article Info

Accepted:

20 September 2020

Available Online:

10 October 2020

\section{Introduction}

Among the different vegetable crops, tomato (Solanum lycopersicum L.) is one of the most popularly cultivated warm season vegetable throughout the world due to its multipurpose use (Triveni et al., 2017). It can also be used as an excellent model crop for study with respect to breeding point of view due to having small diploid genome $(930 \mathrm{Mb}, \mathrm{n}=$ 12) with numerous mapped traits, developed DNA markers and EST clones (Tanksley,
1993). Tomato under target specific selection resulted in increased productivity, but at the same time it has narrowed its genetic basis (Zorzoli et al., 2007) that justified the further study and thorough genetic evaluation of tomato. In spite of all these, major constraints in the diminishing returns of the grower includes varietal fluctuation in the productivity under huge artificial genetic manipulation and contamination leading impure narrow genetic base (Chattopadhyay et al., 2007). It indicated the need for 
exploration for genetic diversity within the available germplasm and utilization of them in conventional cross breeding approach that will be more environmentally safe and practically viable to manifest the advantages in terms of high productivity, uniformity, improved quality, biotic resistance, wider adaptation, earliness and chance for combining of desirable different characters from contrasting parents or inbred (Tesi et al., 1970). In order to select suitable parents and for success of hybridization programme the information pertaining with respect to genetic diversity, its nature and magnitude is important (Fernie et al., 2006; Gami et al., 2010). Line $\times$ Tester design which is basically an extension of top cross is most reliable in estimating combining ability and gene action, offers an advantage in assessment of large number of lines by attempting minimum number of crosses and provides overall genetic background of materials employed under investigation to the breeder (Kempthorne, 1957). Heterosis phenomenon due to its enhanced expression for the manifestation of different characters in both (positive and negative) direction helps in a crop improvement, therefore heterosis breeding serves as an important tool in tomato (Fageria et al., 2001; Duhan et al., 2005; Thakur et al., 2004).

\section{Materials and Methods}

A total of 11 genotypes comprising of 7 lines viz., Arka Abha, Arka Vikas, Arka Saurabh, Arka Meghali, Arka Alok, Arka Ahuti and Arka Ashish sourced from IIHR, Bengaluru, Karnataka as females and 4 widely diversified and highly adoptable testers viz., Patharkutchi, Berika, Alisa Craig received from Bidhan Chandra Krishi Viswavidyalaya, West Bengal and Pusa Ruby from Bihar Agricultural University, Sabour, Bihar as males were employed in Line $\times$ Tester mating model with simple Randomized Block Design
(RBD) at a spacing of $60 \mathrm{~cm} \times 60 \mathrm{~cm}$ to fulfill the objectives of the study. Pusa Ruby was considered as a standard parent. The data was recorded on different yield contributing characters viz., flower/truss, fruit/truss, truss/plant, fruit/plant, polar diameter $(\mathrm{cm})$, equatorial diameter $(\mathrm{cm})$, pericarp thickness $(\mathrm{mm})$, locule number and fruit weight $(\mathrm{g})$ as per published descriptors of IBPGR, Rome and NBPGR, New Delhi. The combining ability analysis was done using the line $x$ tester method described by Kempthorne (1957). Significance for heterosis was tested as per the method suggested by Wynne et al., (1970).

\section{Results and Discussion}

Analysis of variance for lines, testers and hybrids for set of yield contributing characters were represented in Table 1. In the present investigation, mean sum of square for replication was insignificant for irrespective of characters studied indicated that within the selected parents under the experiment were highly homogenous. Variance due to genotypes was partitioned into parents, hybrids and parent vs. hybrids components. The presence of high genetic diversity among the parents used and their derived off-springs was depicted by the highly significant variances due to the genotypes, parents and hybrids for the truss/plant, fruit/plant, fruit weight, polar diameter and pericarp thickness. Highly significant variance due to parents $v s$. hybrids for the fruits/plant, fruit weight, polar diameter, pericarp thickness and locule number suggested the manifestation of heterotic effects for these characters. The existence of enormous diversity among the parents and significant contribution of hybrids derived from them towards specific combining ability was demonstrated by the highly significant variances due to line $v s$. testers for the truss/plant, fruits/plant, fruit weight and pericarp thickness. Highly 
significant variances due to lines for the truss/plant, fruit/plant, fruit weight, pericarp thickness and locule number and due to testers for the fruit/truss, truss/plant, fruit/plant, fruit weight, polar diameter, equatorial diameter and pericarp thickness revealed their contribution towards general combining ability. Insignificant variance due to genotypes for flower/truss and fruit/truss, due to parents for fruits/truss and locule number, due to hybrids for flower/truss, fruit/truss and locule number, due to parents vs. hybrids for flower/truss, fruit/truss, truss/plant and equatorial diameter, due to lines for flower/truss, fruit/truss, polar diameter and equatorial diameter, due to testers for flower per truss and locule number and due to lines vs. testers for flower/truss, fruit/truss, polar diameter, equatorial diameter and locule number were observed.

For designing the effective advanced breeding strategy, the knowledge about the gene action in manifestation of yield contributing traits are enormous important. Comparative assessment of the additive and non-additive gene effect was illustrated by the predictability ratio i.e., the proportion of additive genetic variance to the total genetic variance. In the investigation the magnitude of predictability ratio for all the traits under study was very less than unity (Table 2) indicated preponderance of non-additive gene action for the inheritance of all the traits. Regarding the contribution of lines, testers and lines $\times$ testers towards the total variation, very much diversified response was evident (Table 2). It was observed that there was predominant contribution of lines $\mathrm{x}$ testers interaction towards the total variance for maximum numbers of characters viz., flower per truss, truss per plant, fruit per plant, pericarp thickness. More or less equal contribution of testers and line $\mathrm{x}$ testers interaction towards the total variation was evident for fruit per truss, fruit weight, polar and equatorial diameter of the fruit. There was predominant contribution of lines and lines $\mathrm{x}$ testers interaction for locule number per fruit. These findings found ample support from the earlier workers (Raj et al., 2017 for Pericarp thickness, Savale and Patel, 2017 for fruits/plant, Sherpa et al., 2014 for Locule number/fruit, Shankar et al., 2013 for Fruits/truss and Fruit weight, Yadav et al., 2013 for polar diameter, Shende et al., 2012 for Truss/plant and Equatorial diameter and Sekhar et al., 2010 for Flower/truss).

Per se performance data of parents represented in Table 3 revealed that, Arka Ashish showed the maximum values for number of truss per plant (9.48), polar diameter $(6.69 \mathrm{~cm})$ and pericarp thickness $(8.48 \mathrm{~mm})$. Arka Vikas showed the maximum value for number of fruit per truss (5.32), number of fruit per plant (47.34). Whereas, Arka Saurabh exhibited highest magnitude for number of flower per truss (6.64). Among the testers highest magnitude towards desired direction was recorded in Berika for fruit weight $(107.92 \mathrm{~g})$, polar diameter $(6.89 \mathrm{~cm})$, equatorial diameter $(5.45 \mathrm{~cm})$, pericarp thickness $(8.54 \mathrm{~mm})$ and locule number (2.0) and by Patharkutchi for fruit per truss (5.45), truss per plant (8.21) and fruit per plant (44.72). Comparison between the average magnitude in desired direction between lines and testers indicated that among the 9 yield contributing characters studied, average performance of lines was higher than the testers for 6 characters that highly justified the selection of parents for investigation as well as greater genetic potentiality to develop the hybrids. It was depicted in the Table 4 that Per se performance considering the Arka Alok hybrids altogether was highest for the characters viz., number of flower per truss, number of fruit per truss, fruit weight and equatorial diameter. Whereas, Arka Saurabh hybrids showed the maximum average value for number of fruit and truss per plant and 
locule number per fruit. Mean pericarp thickness was recorded to be the highest at desired direction in the Arka Meghali hybrids. Average performance of Arka Ashish hybrids for polar diameter displayed highest. Considering the individual $\mathrm{Per}$ se performance separately it was found that the for flower per truss the maximum value recorded in Arka Saurabh $\times$ Berika (6.34). Similarly, for number of fruit per truss (5.56) and fruit weight (147.66 g) was recorded to be the highest in Arka Alok $\times$ Berika. Whereas, number of truss per plant (15.12) and fruit per plant (65.57) was recorded maximum in Arka Saurabh $\times$ Alisa Craig. Regarding the polar diameter $(6.39 \mathrm{~cm})$ and locule number per fruit (2.01) was recorded maximum (desired path) in Arka Ahuti $\times$ Berika. For the equatorial diameter the highest value was recorded in Arka Alok $\times$ Patharkutchi (6.71 $\mathrm{cm})$. Whereas, for pericarp thickness it was observed that Arka Alok $\times$ Alisa Craig (9.09 $\mathrm{mm}$ ) recorded the maximum magnitude.

Comparison between the average magnitude at desired direction between lines and testers together with the average performance of hybrids indicated that there was an unregulated manifestation among the hybrids in comparison to average performance of their parents suggested the importance of heterosis for manifestation of most of the characters.

The general combining ability (GCA) effect for all yield contributing characters were represented in Table 3 for lines as well as testers separately. The highest positive GCA effects among lines were recorded in Arka Alok for flower per truss (0.53), fruits per truss (0.67), fruit weight (21.60) and equatorial diameter (0.64). Whereas, in Arka Saurabh for truss per plant (1.82), fruits per plant (8.31) and locule number (0.60), Arka Ashish (0.53) for polar diameter, Arka Meghali (1.33) for pericarp thickness. Similarly, among testers Berika for flower per truss $(0.36)$, fruit per truss $(0.35)$, fruit weight (24.48 and polar diameter (0.85), Alisa Craig for truss per plant (1.77), fruit per plant (9.69) and pericarp thickness (0.46), Pusa Ruby for equatorial diameter (0.38) and Patharkutchi for locule number (0.41) showed maximum positive GCA effects. Among lines Arka Alok, Arka Saurabh, Arka Vikas and Arka Meghali identified as good with general combining ability. Similarly, among testers Berika and Alisa Craig possess good general combining ability.

The specific combining ability effect (SCA) for all the studied characters was represented in Table 5. Regarding flower per truss highest values were recorded in Arka Ahuti $\times$ Alisa Craig (0.89) followed by Arka Alok $\times$ Patharkutchi (0.72) and Arka Saurabh $\times$ Berika (0.46). Similarly, for fruit per truss was recorded in Arka Abha $\times$ Alisa Craig (0.79) followed by Arka Saurabh $\times$ Berika (0.72) and Arka Ahuti $\times$ Patharkutchi (0.42), for the truss per plant was noted in Arka Saurabh $\times$ Alisa Crag (4.00) followed by Arka Ahuti $\times$ Alisa Craig (2.97) and Arka Abha $\times$ Berika (1.86), Arka Ahuti $\times$ Alisa Craig (16.97) followed by Arka Saurabh $\times$ Alisa Craig (14.17) and Arka Meghali $\times$ Patharkutchi (8.86) for fruit per plant. In case of individual mature ripe fruit weight Arka Ahuti $\times$ Pusa Ruby (35.42) followed by Arka Alok $\times$ Patharkutchi (30.43) and Arka Ashish $\times$ Alisa Craig (26.57) preformed the maximum. Regarding the polar diameter, maximum recorded in Arka Abha $\times$ Alisa Craig (0.80) followed by Arka Ahuti $\times$ Berika (0.70) and Arka Meghali $\times$ Alisa Craig (0.66), for equatorial diameter in Arka Abha $\times$ Alisa Craig (0.85) followed by Arka Alok $\times$ Patharkutchi (0.83) and Arka Ahuti $\times$ Pusa Ruby (0.71), for pericarp thickness, in Arka Abha $\times$ Alisa Craig (1.96) followed by Arka Abha $\times$ Pusa Ruby (1.83) and Arka Vikas $\times$ Patharkutchi (1.76) and for locule number of fruit was recorded in Arka Ashish $\times$ Berika 
(0.86) followed by Arka Saurabh $\times$ Alisa Crag (0.63) and Arka Vikas $\times$ Berika (0.62).

Arka Saurabh $\times$ Alisa Craig, Arka Ashish $\times$ Berika and Arka Vikas $\times$ Berika crosses identified as best with good specific combining ability which may be due to good $\times$ good and poor $\times$ good cross combinations of GCA effects of parents.

The range and magnitude of the heterosis estimated over mid parent (relative heterosis), better parent (heterobeltiosis) and standard parent considering Pusa Ruby as check variety (Standard heterosis) have been presented in Table 6. For flower per truss the highest magnitude of average heterosis was observed in Arka Alok $\times$ Pusa Ruby (20.07\%), heterobeltiosis in Arka Alok $\times$ Berika (16.92\%) and standard heterosis in Arka Saurabh $\times$ Berika (55.77\%). The cross Arka Abha $\times$ Alisa Craig displayed maximum average heterosis $(30.43 \%)$ for fruit per truss and similarly Arka Alok $\times$ Berika exhibited maximum better parent heterosis $(27.04 \%)$ and standard parent heterosis $(59.92 \%)$ for the same character.

Table.1 Analysis of variance for parents and hybrids for yield contributing characters

\begin{tabular}{|c|c|c|c|c|c|c|c|c|c|}
\hline \multirow{2}{*}{$\begin{array}{c}\text { Source of variation } \\
\text { (d.f) }\end{array}$} & \multicolumn{9}{|c|}{ Mean Sum of Square for Parents and Hybrids for different yield contributing characters } \\
\hline & $\begin{array}{l}\text { Flower } \\
\text { / truss }\end{array}$ & $\begin{array}{l}\text { Fruit/ } \\
\text { truss }\end{array}$ & $\begin{array}{l}\text { Truss/ } \\
\text { plant }\end{array}$ & $\begin{array}{c}\text { Fruit/ } \\
\text { plant }\end{array}$ & $\begin{array}{c}\text { Fruit } \\
\text { weight (g) }\end{array}$ & $\begin{array}{c}\text { Polar } \\
\text { diameter } \\
(\mathbf{c m})\end{array}$ & $\begin{array}{l}\text { Equatoria } \\
\text { I diameter } \\
\text { (cm) }\end{array}$ & $\begin{array}{c}\text { Pericarp } \\
\text { thickness } \\
(\mathbf{m m})\end{array}$ & $\begin{array}{c}\text { Locule } \\
\text { numbe } \\
\mathbf{r}\end{array}$ \\
\hline Replication (2) & 0.003 & 0.012 & 0.0005 & 0.878 & 0.046 & 0.003 & 0.003 & 0.005 & 0.002 \\
\hline Genotypes (38) & 1.372 & 1.176 & $11.05 * *$ & $306.44 * *$ & $2147.96 * *$ & $2.469 * *$ & $1.744 *$ & $9.626 * *$ & $1.812^{* *}$ \\
\hline Parents (10) & $2.225^{*}$ & 1.638 & $2.188 *$ & $166.76 * *$ & $535.09 * *$ & $3.338 * *$ & 0.679 & $9.125 * *$ & 1.882 \\
\hline Hybrids (27) & 1.083 & 1.043 & $14.60 * *$ & $368.43 * *$ & $2747.44 * *$ & $2.089 * *$ & $2.175 * *$ & $9.988 * *$ & 1.596 \\
\hline Parents vs Hybrids (1) & 0.626 & 0.155 & 3.926 & $29.27 * *$ & $2091.02 * *$ & $4.048 *$ & 0.766 & $4.838 *$ & $6.880 *$ \\
\hline Lines $(6)$ & 1.260 & 1.530 & $10.80 * *$ & $364.09 * *$ & $2335.95 * *$ & 2.130 & 1.922 & $15.65 * *$ & $3.091 * *$ \\
\hline Testers (3) & 2.335 & $2.882 *$ & $33.26 * *$ & $984.68 * *$ & $9769.88 * *$ & $6.886 * *$ & $6.392 * *$ & $5.153 * *$ & 1.861 \\
\hline Lines vs Testers (18) & 0.815 & 0.575 & $12.75 * *$ & $267.17 * *$ & $1714.19 * *$ & 1.275 & 1.556 & $8.906 * *$ & 1.056 \\
\hline Error (76) & 0.003 & 0.002 & 0.003 & 0.268 & 0.247 & 0.002 & 0.002 & 0.011 & 0.002 \\
\hline
\end{tabular}

$* *=$ Significance at $1 \%$ level; $*=$ Significance at $5 \%$ level

Table.2 Estimates of genetic components and proportional contribution to variance for yield contributing characters

\begin{tabular}{|c|c|c|c|c|c|c|c|c|c|}
\hline $\begin{array}{l}\text { Components of } \\
\text { genetic variation }\end{array}$ & $\begin{array}{l}\text { Flower } \\
\text { / truss }\end{array}$ & $\begin{array}{c}\text { Fruit/ } \\
\text { truss }\end{array}$ & $\begin{array}{c}\text { Truss } \\
/ \\
\text { plant }\end{array}$ & $\begin{array}{l}\text { Fruit/ } \\
\text { plant }\end{array}$ & $\begin{array}{c}\text { Fruit } \\
\text { weight } \\
\text { (g) }\end{array}$ & $\begin{array}{c}\text { Polar } \\
\text { diameter } \\
(\mathrm{cm})\end{array}$ & $\begin{array}{c}\text { Equatoria } \\
\text { I } \\
\text { diameter } \\
(\mathrm{cm})\end{array}$ & $\begin{array}{c}\text { Pericarp } \\
\text { thickness } \\
(\mathbf{m m})\end{array}$ & $\begin{array}{l}\text { Locule } \\
\text { number }\end{array}$ \\
\hline$\sigma^{2}$ GCA & 0.006 & 0.010 & 0.041 & 2.25 & 22.96 & 0.018 & 0.014 & 0.024 & 0.012 \\
\hline$\sigma^{2} A$ & 0.012 & 0.021 & 0.082 & 4.50 & 45.92 & 0.036 & 0.027 & 0.048 & 0.024 \\
\hline$\sigma^{2} \operatorname{SCA}\left(\sigma^{2} D\right)$ & 0.467 & 0.515 & 6.16 & 171.21 & 1451.29 & 1.078 & 1.046 & 3.209 & 0.623 \\
\hline Predictability ratio & 0.025 & 0.039 & 0.013 & 0.026 & 0.031 & 0.033 & 0.026 & 0.015 & 0.037 \\
\hline \multicolumn{10}{|c|}{ Proportional contribution to the total variance $(\%)$} \\
\hline Lines & 25.85 & 32.60 & 16.44 & 21.96 & 18.94 & 22.66 & 19.64 & 34.82 & 43.00 \\
\hline Testers & 23.96 & 30.69 & 25.31 & 29.70 & 39.51 & 36.63 & 32.66 & 5.73 & 12.94 \\
\hline Line $\mathbf{X}$ Tester & 50.19 & 36.72 & 58.24 & 48.34 & 41.59 & 40.70 & 47.70 & 59.44 & 44.06 \\
\hline
\end{tabular}


Table.3 GCA effect and average performance of Lines and Testers for yield contributing characters

\begin{tabular}{|c|c|c|c|c|c|c|c|c|c|c|}
\hline \multicolumn{2}{|l|}{ Genotype } & $\begin{array}{c}\text { Flower/ } \\
\text { truss }\end{array}$ & $\begin{array}{c}\text { Fruit/ } \\
\text { truss }\end{array}$ & $\begin{array}{l}\text { Truss/ } \\
\text { plant }\end{array}$ & $\begin{array}{l}\text { Fruit/ } \\
\text { plant }\end{array}$ & $\begin{array}{c}\text { Fruit } \\
\text { weight } \\
\text { (g) }\end{array}$ & $\begin{array}{c}\text { Polar } \\
\text { diameter } \\
(\mathbf{c m})\end{array}$ & $\begin{array}{l}\text { Equatorial } \\
\text { diameter } \\
\text { (cm) }\end{array}$ & $\begin{array}{c}\text { Pericarp } \\
\text { thickness } \\
(\mathbf{m m})\end{array}$ & $\begin{array}{l}\text { Locule } \\
\text { number }\end{array}$ \\
\hline \multicolumn{11}{|c|}{ Lines } \\
\hline \multirow[t]{2}{*}{ Arka Alok (L1) } & gca & $0.53 * *$ & $0.67 * *$ & $0.18 * *$ & $5.58 * *$ & $21.60 * *$ & $0.30 * *$ & $0.64 * *$ & $0.43 * *$ & $0.55 * *$ \\
\hline & $\mu$ & 5.23 & 4.19 & 7.22 & 30.23 & 123.60 & 6.08 & 5.88 & 8.14 & 4.39 \\
\hline \multirow[t]{2}{*}{ Arka Abha (L2) } & gca & $-0.30 * *$ & $-0.05^{* *}$ & $-0.40 * *$ & $-2.52 * *$ & $-2.44 * *$ & $-0.36 * *$ & $0.17 * *$ & $-0.77 * *$ & $0.04 * *$ \\
\hline & $\mu$ & 4.30 & 3.27 & 8.06 & 26.34 & 92.67 & 4.28 & 5.30 & 4.65 & 4.66 \\
\hline \multirow[t]{2}{*}{ Arka Ashish (L3) } & gca & $0.14 * *$ & $0.06 * *$ & $-0.56 * *$ & $-2.18 * *$ & $-3.32 * *$ & $0.53 * *$ & $-0.29 * *$ & $0.93 * *$ & $-0.47 * *$ \\
\hline & $\mu$ & 6.02 & 4.06 & 9.48 & 38.51 & 107.08 & 6.69 & 4.97 & 8.48 & 3.37 \\
\hline \multirow[t]{2}{*}{ Arka Ahuti (L4) } & gca & $-0.25 * *$ & $-0.09 * *$ & $0.52 * *$ & $2.23 * *$ & $-9.66 * *$ & 0.03* & $-0.51 * *$ & $0.41 * *$ & $-0.79 * *$ \\
\hline & $\mu$ & 4.88 & 3.78 & 8.73 & 33.04 & 109.65 & 6.02 & 5.71 & 7.15 & 3.37 \\
\hline \multirow[t]{2}{*}{ Arka Meghali (L5) } & gca & $-0.36 * *$ & $-0.55^{* *}$ & $-0.57 * *$ & $-6.29 * *$ & $13.27 * *$ & $0.30 * *$ & $0.15 * *$ & $1.33 * *$ & $-0.10 * *$ \\
\hline & $\mu$ & 4.95 & 3.90 & 7.46 & 29.08 & 111.10 & 4.99 & 5.71 & 5.48 & 3.68 \\
\hline \multirow[t]{2}{*}{ Arka Vikas (L6) } & gca & 0.02 & $-0.07 * *$ & $-1.00^{* *}$ & $-5.13 * *$ & $0.90 * *$ & $-0.11 * *$ & $0.18 * *$ & $-0.29 * *$ & $0.16 * *$ \\
\hline & $\mu$ & 6.41 & 5.32 & 8.89 & 47.34 & 109.26 & 4.52 & 5.63 & 4.07 & 4.32 \\
\hline \multirow{2}{*}{$\begin{array}{c}\text { Arka Saurabh } \\
\text { (L7) }\end{array}$} & gca & $0.21 * *$ & 0.04* & $1.82 * *$ & $8.31 * *$ & $-20.35 * *$ & $-0.68^{* *}$ & $-0.36 * *$ & $-2.04 * *$ & $0.60 * *$ \\
\hline & $\mu$ & 6.64 & 4.81 & 7.30 & 35.11 & 96.76 & 4.68 & 5.80 & 5.70 & 3.35 \\
\hline \multicolumn{2}{|l|}{ S.E. $\left(g_{1}\right)$} & 0.015 & 0.014 & 0.015 & 0.149 & 0.143 & 0.012 & 0.013 & 0.031 & 0.013 \\
\hline \multicolumn{11}{|c|}{ Testers } \\
\hline \multirow[t]{2}{*}{ Patharkuchi (T1) } & gca & $-0.29 * *$ & $-0.30 * *$ & $-1.00 * *$ & $-6.37 * *$ & $-2.52 * *$ & $-0.22 * *$ & $0.06 * *$ & $-0.30 * *$ & $0.41 * *$ \\
\hline & $\mu$ & 6.06 & 5.45 & 8.21 & 44.72 & 77.50 & 3.98 & 4.27 & 3.99 & 4.72 \\
\hline \multirow[t]{2}{*}{ Berika (T2) } & gca & $0.36 * *$ & $0.35 * *$ & $-0.78 * *$ & $-1.06^{* * *}$ & $24.48 * *$ & $0.85^{* *}$ & $0.35 * *$ & $-0.54 * *$ & $-0.30 * *$ \\
\hline & $\mu$ & 5.42 & 4.38 & 7.83 & 34.29 & 107.92 & 6.89 & 5.45 & 8.54 & 2.00 \\
\hline \multirow[t]{2}{*}{ Alisa Craig (T3) } & gca & $0.21 * *$ & $0.29 * *$ & $1.77 * *$ & $9.69 * *$ & $-27.55 * *$ & $-0.38 * *$ & $-0.80 * *$ & $0.46 * *$ & $-0.11 * *$ \\
\hline & $\mu$ & 6.17 & 5.08 & 7.56 & 38.39 & 95.57 & 5.42 & 5.06 & 6.77 & 3.42 \\
\hline \multirow[t]{2}{*}{ Pusa Ruby (T4) } & gca & $-0.28 * *$ & $-0.34 * *$ & 0.01 & $-2.25 * *$ & $5.58 * *$ & $-0.26 * *$ & $0.38 * *$ & $0.38 * *$ & 0.001 \\
\hline & $\mu$ & 4.07 & 3.48 & 6.54 & 22.73 & 84.40 & 3.99 & 5.19 & 4.72 & 4.12 \\
\hline \multicolumn{2}{|l|}{ S.E. $\left(g_{t}\right)$} & 0.018 & 0.011 & 0.011 & 0.113 & 0.108 & 0.009 & 0.010 & 0.023 & 0.010 \\
\hline
\end{tabular}

$* *=$ Significance at $1 \%$ level $; *=$ Significance at $5 \%$ level 
Table.4 Average mean performance of the hybrids for the yield contributing characters

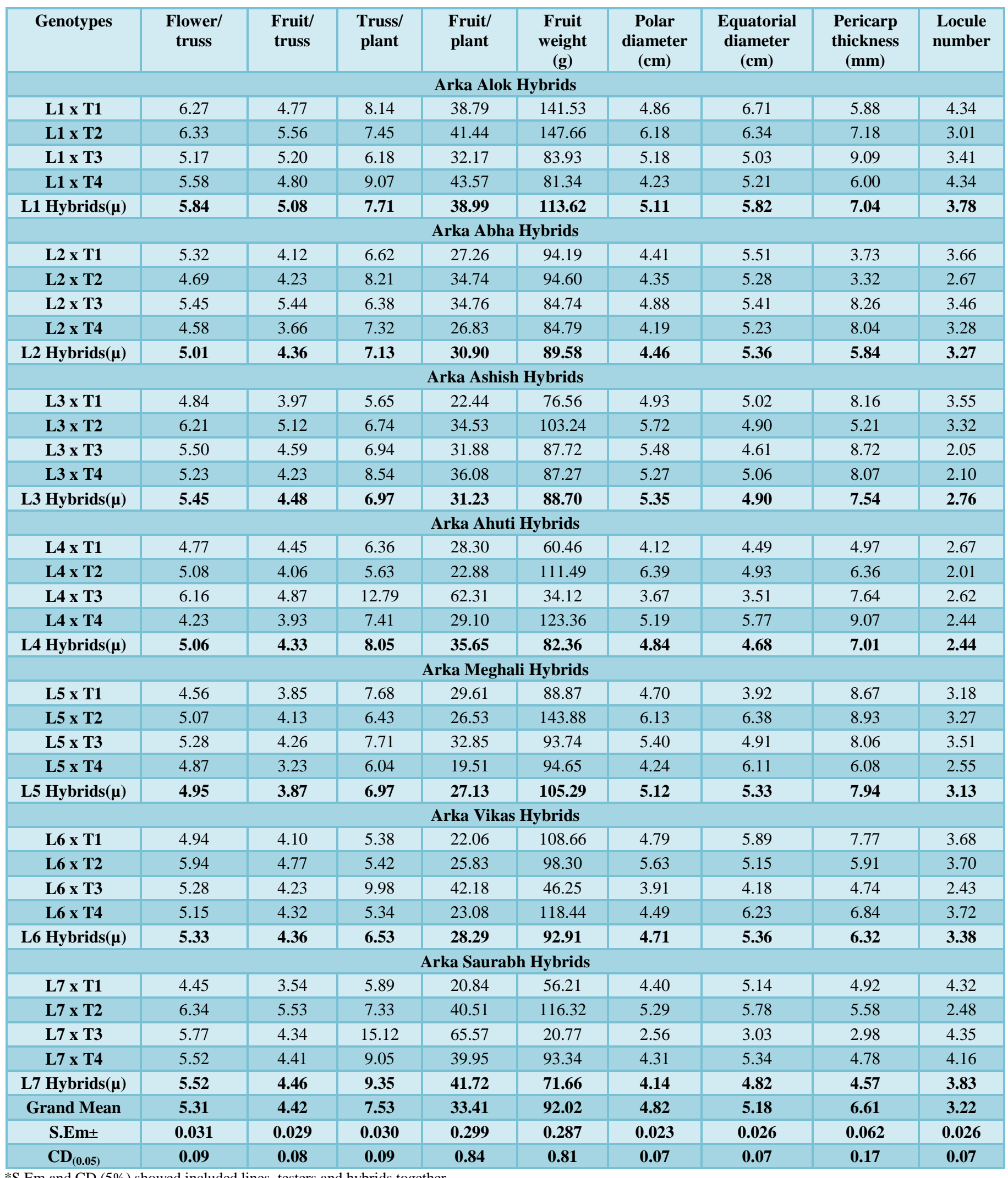


Table.5 SCA effect for Hybrids for yield contributing characters

\begin{tabular}{|c|c|c|c|c|c|c|c|c|c|}
\hline Genotype & $\begin{array}{c}\text { Flower/ } \\
\text { truss }\end{array}$ & $\begin{array}{c}\text { Fruit/ } \\
\text { truss }\end{array}$ & $\begin{array}{l}\text { Truss/ } \\
\text { plant }\end{array}$ & $\begin{array}{l}\text { Fruit/ } \\
\text { plant }\end{array}$ & $\begin{array}{c}\text { Fruit } \\
\text { weight }(\mathrm{g})\end{array}$ & $\begin{array}{c}\text { Polar } \\
\text { diameter } \\
(\mathbf{c m})\end{array}$ & $\begin{array}{l}\text { Equatoria } \\
\text { I diameter } \\
\text { (cm) }\end{array}$ & $\begin{array}{c}\text { Pericarp } \\
\text { thicknes } \\
\text { s (mm) }\end{array}$ & $\begin{array}{c}\text { Locule } \\
\text { number }\end{array}$ \\
\hline L1 x T1 & $0.72 * *$ & -0.01 & $1.42 * *$ & $6.17 * *$ & $30.43 * *$ & -0.04 & $0.83 * *$ & $-0.85^{* *}$ & $0.16^{* *}$ \\
\hline $\mathbf{L 1} \times \mathbf{T} 2$ & $0.14 * *$ & $0.12 * *$ & $0.53^{* *}$ & $3.51 * *$ & $9.56^{* *}$ & $0.22 * *$ & $0.16^{* *}$ & $0.68^{* *}$ & $-0.46^{* *}$ \\
\hline L1 x T3 & $-0.88 * *$ & $-0.17 * *$ & $-3.30 * *$ & $-16.51 * *$ & $-2.14 * *$ & $0.45^{* *}$ & 0.01 & $1.59^{* *}$ & $-0.26 * *$ \\
\hline L1 x T4 & 0.03 & 0.06 & $1.35^{* *}$ & $6.83^{* *}$ & $-37.86^{* *}$ & $-0.63^{* *}$ & $-1.00 * *$ & $-1.42 * *$ & $0.56 * *$ \\
\hline L2 x T1 & $0.59 * *$ & 0.06 & $0.49 * *$ & $2.73 * *$ & $7.13 * *$ & $0.17 * *$ & $0.09 * *$ & $-1.80 * *$ & -0.01 \\
\hline $\mathrm{L} 2 \times \mathrm{T} 2$ & $-0.68 * *$ & $-0.48 * *$ & $1.86^{* *}$ & $4.91 * *$ & $-19.46^{* *}$ & $-0.96^{* *}$ & $-0.43^{* *}$ & $-1.98 * *$ & $-0.29 * *$ \\
\hline L2 x T3 & $0.23 * *$ & $0.79 * *$ & $-2.52 * *$ & $-5.83 * *$ & $22.71 * *$ & $0.80^{* *}$ & $0.85^{* *}$ & $1.96^{* *}$ & $0.30 * *$ \\
\hline L2 x T4 & $-0.14 * *$ & $-0.37 * *$ & $0.18^{* *}$ & $-1.81 * *$ & $-10.37 * *$ & -0.01 & $-0.51 * *$ & $1.83^{* *}$ & 0.01 \\
\hline L3 x T1 & $-0.32 * *$ & $-0.20 * *$ & $-0.32 * *$ & $-2.42 * *$ & $-9.62 * *$ & $-0.20 * *$ & $0.06^{*}$ & $0.92 * *$ & $0.39 * *$ \\
\hline L3 x T2 & $0.40 * *$ & $0.29 * *$ & $0.56^{* *}$ & $4.36 * *$ & $-9.94 * *$ & $-0.48 * *$ & $-0.35 * *$ & $-1.80 * *$ & $0.86 * *$ \\
\hline L3 $x$ T3 & $-0.16 * *$ & $-0.18^{* *}$ & $-1.80 * *$ & $-9.04 * *$ & $26.57 * *$ & $0.51 * *$ & $0.51 * *$ & $0.72 * *$ & $-0.60^{* * *}$ \\
\hline L3 x T4 & $0.07 *$ & $0.08 * *$ & $1.56^{* *}$ & $7.10^{* *}$ & $-7.01 * *$ & $0.18 * *$ & $-0.22 * *$ & $0.15^{*}$ & $-0.66 * *$ \\
\hline L4 x T1 & 0.004 & $0.42 * *$ & $-0.69 * *$ & $-0.98 * *$ & $-19.38 * *$ & $-0.51 * *$ & $-0.25 * *$ & $-1.74 * *$ & $-0.17 * *$ \\
\hline $\mathrm{L} 4 \times \mathrm{T} 2$ & $-0.34 * *$ & $-0.62 * *$ & $-1.63 * *$ & $-11.71 * *$ & $4.65^{* *}$ & $0.70 * *$ & $-0.10 * *$ & -0.11 & $-0.12^{* *}$ \\
\hline L4 x T3 & $0.89 * *$ & $0.26^{* *}$ & $2.97 * *$ & $16.97 * *$ & $-20.69 * *$ & $-0.79 * *$ & $-0.37 * *$ & $0.17 * *$ & $0.29 * *$ \\
\hline L4 x T4 & $-0.55^{* *}$ & $-0.07 *$ & $-0.65 * *$ & $-4.29 * *$ & $35.42 * *$ & $0.61 * *$ & $0.71 * *$ & $1.68^{* * *}$ & 0.002 \\
\hline L5 x T1 & $-0.10 * *$ & $0.29 * *$ & $1.72 * *$ & $8.86^{* *}$ & $-13.90 * *$ & $-0.20 * *$ & $-1.47 * *$ & $1.04 * *$ & $-0.35^{* *}$ \\
\hline L5 x T2 & $-0.24 * *$ & $-0.09 * *$ & $0.25^{* *}$ & 0.47 & $14.11 * *$ & $0.16^{* *}$ & $0.69 * *$ & $1.53^{* *}$ & $0.44 * *$ \\
\hline L5 x T3 & $0.13^{* *}$ & $0.11 * *$ & $-1.03 * *$ & $-3.96 * *$ & $16.01 * *$ & $0.66 * *$ & $0.38 * *$ & $-0.34 * *$ & $0.49 * *$ \\
\hline L5 $\times$ T4 & $0.21 * *$ & $-0.30 * *$ & $-0.94 * *$ & $-5.36 * *$ & $-16.22 * *$ & $-0.62 * *$ & $0.39 * *$ & $-2.23 * *$ & -0.58 ** \\
\hline L6 x T1 & $-0.11 * *$ & 0.05 & $-0.15 * *$ & 0.14 & $18.26^{* *}$ & $0.30 * *$ & $0.47 * *$ & $1.76^{* *}$ & $-0.11^{* *}$ \\
\hline L6 x T2 & $0.25^{* *}$ & $0.06^{*}$ & $-0.33 * *$ & $-1.40 * *$ & $-19.09 * *$ & $0.07 * *$ & $-0.57 * *$ & $0.13^{*}$ & $0.62 * *$ \\
\hline L6 x T3 & $-0.26 * *$ & $-0.41 * *$ & $1.68 * *$ & $4.20 * *$ & $-19.11 * *$ & $-0.42 * *$ & $-0.38 * *$ & $-2.04 * *$ & $-0.85^{* *}$ \\
\hline L6 $x$ T4 & $0.11^{* *}$ & $0.30 * *$ & $-1.20 * *$ & $-2.95^{* *}$ & $19.94 * *$ & 0.05 & $0.49 * *$ & $0.14^{*}$ & $0.33 * *$ \\
\hline L7 x T1 & $-0.78 * *$ & $-0.61 * *$ & $-2.46^{* *}$ & $-14.51 * *$ & $-12.93 * *$ & $0.48 * *$ & $0.26 * *$ & $0.66^{* *}$ & $0.09 * *$ \\
\hline L7 x T2 & $0.46 * *$ & $0.72 * *$ & $-1.23 * *$ & -0.14 & $20.17 * *$ & $0.30 * *$ & $0.61 * *$ & $1.55^{* *}$ & $-1.05^{* *}$ \\
\hline L7 x T3 & 0.04 & $-0.40 * *$ & $4.00^{* *}$ & $14.17 * *$ & $-23.34 * *$ & $-1.21 * *$ & $-1.00 * *$ & $-2.05 * *$ & $0.63 * *$ \\
\hline L7 x T4 & $0.28 * *$ & $0.29 * *$ & $-0.30 * *$ & 0.49 & $16.10^{* *}$ & $0.42 * *$ & $0.13^{* *}$ & $-0.16^{*}$ & $0.33 * *$ \\
\hline S.E. $\left(s_{i j}\right)$ & 0.031 & 0.029 & 0.030 & 0.299 & 0.287 & 0.024 & 0.026 & 0.062 & 0.026 \\
\hline
\end{tabular}

$* *=$ Significance at $1 \%$ level; *= Significance at $5 \%$ level 
Table.6 Heterosis (\%) over mid parent, better parent and standard parent for the yield contributing characters

\begin{tabular}{|c|c|c|c|c|c|c|c|c|c|c|}
\hline \multicolumn{2}{|c|}{ Genotype } & \multirow{2}{*}{$\begin{array}{c}\begin{array}{c}\text { Flower/ } \\
\text { truss }\end{array} \\
11.11^{* *}\end{array}$} & \multirow{2}{*}{$\begin{array}{c}\begin{array}{c}\text { Fruit/ } \\
\text { truss }\end{array} \\
-1.07 \\
\end{array}$} & \multirow{2}{*}{$\begin{array}{c}\begin{array}{c}\text { Truss/ } \\
\text { plant }\end{array} \\
5.488^{* *}\end{array}$} & \multirow{2}{*}{$\begin{array}{c}\begin{array}{c}\text { Fruit/ } \\
\text { plant }\end{array} \\
3.51^{* *}\end{array}$} & \multirow{2}{*}{$\begin{array}{c}\begin{array}{c}\text { Fruit } \\
\text { weight (g) }\end{array} \\
\\
\text { 40.76** }^{* *}\end{array}$} & \multirow{2}{*}{$\begin{array}{c}\begin{array}{c}\text { Polar } \\
\text { diameter } \\
(\mathbf{c m})\end{array} \\
-3.41^{* *}\end{array}$} & \multirow{2}{*}{$\begin{array}{c}\begin{array}{c}\text { Equatorial } \\
\text { diameter } \\
(\mathbf{c m})\end{array} \\
32.24 * * \\
\end{array}$} & \multirow{2}{*}{$\begin{array}{c}\begin{array}{c}\text { Pericarp } \\
\text { thickness } \\
(\mathbf{m m})\end{array} \\
-3.00^{* *}\end{array}$} & \multirow{2}{*}{$\begin{array}{c}\begin{array}{c}\text { Locule } \\
\text { number }\end{array} \\
-4.65^{* *}\end{array}$} \\
\hline L1 x T1 & MP & & & & & & & & & \\
\hline & $\mathrm{BP}$ & $3.52 * *$ & $-12.49 * *$ & $-0.89^{*}$ & $-13.26 * *$ & $14.50 * *$ & $-20.07 * *$ & $14.12 * *$ & $-27.72 * *$ & $-7.98 * *$ \\
\hline & SP & $54.05^{* *}$ & $37.20^{* *}$ & $24.53 * *$ & $70.68 * *$ & $67.68 * *$ & $21.70 * *$ & $29.37 * *$ & $24.66^{* *}$ & $5.26 * *$ \\
\hline \multirow[t]{3}{*}{ L1 x T2 } & MP & $18.97 * *$ & $29.81 * *$ & $-0.952 *$ & $28.47 * *$ & $27.55^{* * *}$ & $-4.68 * *$ & $11.92 * *$ & $-13.89 * *$ & $-5.69 * *$ \\
\hline & BP & $16.92 * *$ & $27.04 * *$ & $-4.85 * *$ & $20.87 * *$ & $19.46^{* *}$ & $-10.30 * *$ & $7.83 * *$ & $-15.89 * *$ & $-31.36^{* * *}$ \\
\hline & SP & $55.53^{* *}$ & 59.92** & $13.97 * *$ & $82.34 * *$ & $74.95 * *$ & $54.76 * *$ & $22.24 * *$ & $52.23 * *$ & $-27.00^{* * *}$ \\
\hline \multirow{3}{*}{ L1 x T3 } & MP & $-9.33 * *$ & $12.26^{* *}$ & $-16.291 * *$ & $-6.24 * *$ & $-23.41 * *$ & $-9.86^{* *}$ & $-8.02 * *$ & $21.90 * *$ & $-12.64 * *$ \\
\hline & BP & $-16.25 * *$ & $2.43 * *$ & $-18.17 * *$ & $-16.20 * *$ & $-32.10 * *$ & $-14.75^{* *}$ & $-14.41 * *$ & $11.67 * *$ & $-22.32 * *$ \\
\hline & SP & $27.03 * *$ & $49.57 * *$ & $-5.46 * *$ & $41.55^{* *}$ & -0.56 & $29.72 * *$ & $-3.02 * *$ & $92.72 * *$ & $-17.30^{* * *}$ \\
\hline \multirow{3}{*}{ L1 x T4 } & MP & $20.07 * *$ & $25.30 * *$ & $31.847 * *$ & $64.54 * *$ & $-21.79 * *$ & $-16.02 * *$ & $-5.82 * *$ & $-6.66 * *$ & $1.88^{* *}$ \\
\hline & BP & $6.76^{* *}$ & $14.64 * *$ & $25.64 * *$ & $44.12 * *$ & $-34.20 * *$ & $-30.43 * *$ & $-11.34 * *$ & $-26.29 * *$ & -1.22 \\
\hline & SP & $37.10^{* *}$ & $38.06^{* *}$ & $38.76^{* *}$ & $91.71 * *$ & $-3.63^{* *}$ & $5.93 * *$ & 0.45 & $27.21 * *$ & $5.26 * *$ \\
\hline \multirow[t]{3}{*}{ L2 x T1 } & MP & $2.70 * *$ & $-5.51 * *$ & $-18.640 * *$ & $-23.29 * *$ & $10.70^{* *}$ & $6.69 * *$ & $15.08^{* * *}$ & $-13.69 * *$ & $-21.96 * *$ \\
\hline & $\mathrm{BP}$ & $-12.22 * *$ & $-24.42 * *$ & $-19.37 * *$ & $-39.06 * *$ & $1.63 * *$ & $2.96 * *$ & $3.83 * *$ & $-19.84 * *$ & $-22.46^{* * *}$ \\
\hline & SP & $30.71 * *$ & $18.50^{* *}$ & $1.27 *$ & $19.95^{* *}$ & $11.60 * *$ & $10.43 * *$ & $6.23 * *$ & $-20.92 * *$ & $-11.24 * *$ \\
\hline \multirow[t]{3}{*}{ L2 x T2 } & MP & $-3.50 * *$ & $10.77 * *$ & $3.250 * *$ & $14.60 * *$ & $-5.69 * *$ & $-22.10 * *$ & $-1.83 * *$ & $-49.61 * *$ & $-19.72 * *$ \\
\hline & BP & $-13.48 * *$ & $-3.28 * *$ & $1.78^{* *}$ & 1.32 & $-12.35^{* *}$ & $-36.85^{* *}$ & $-3.12 * *$ & $-61.07 * *$ & $-42.63 * *$ \\
\hline & SP & $15.23 * *$ & $21.67 * *$ & $25.60 * *$ & $52.86^{* *}$ & $12.08 * *$ & $8.93 * *$ & $1.80^{*}$ & $-29.61 * *$ & $-35.25 * *$ \\
\hline \multirow[t]{3}{*}{ L2 x T3 } & MP & $4.04 * *$ & $30.43 * *$ & $-18.267 * *$ & $7.39 * *$ & $-9.97 * *$ & 0.65 & $4.34 * *$ & $44.52 * *$ & $-14.32 * *$ \\
\hline & BP & $-11.77 * *$ & $7.15 * *$ & $-20.84 * *$ & $-9.46 * *$ & $-11.33 * *$ & $-9.90 * *$ & $1.95 * *$ & $21.90 * *$ & $-25.75^{* *}$ \\
\hline & SP & $33.91 * *$ & $56.47 * *$ & $-2.40 * *$ & $52.95 * *$ & 0.40 & 22.20 ** & $4.31 * *$ & $75.12 * *$ & $-16.09 * *$ \\
\hline \multirow[t]{3}{*}{ L2 x T4 } & MP & $9.56 * *$ & $8.65^{* *}$ & 0.274 & $9.35 * *$ & $-4.23 * *$ & $1.25^{*}$ & -0.29 & $71.68 * *$ & $-25.39 * *$ \\
\hline & BP & $6.67 * *$ & $5.37 * *$ & $-9.22 * *$ & 1.85 & $-8.51 * *$ & $-2.18 * *$ & $-1.38 * *$ & $70.53 * *$ & $-29.69 * *$ \\
\hline & SP & $12.53^{* *}$ & $5.27 * *$ & $11.98^{* *}$ & $18.05^{* * *}$ & 0.46 & $4.93 * *$ & 0.83 & $70.46^{* * *}$ & $-20.45^{* *}$ \\
\hline \multirow[t]{3}{*}{ L3 x T1 } & MP & $-19.81 * *$ & $-16.41 * *$ & $-36.172 * *$ & $-46.08 * *$ & $-17.04 * *$ & $-7.59 * *$ & $8.66 * *$ & $30.82 * *$ & $-12.20 * *$ \\
\hline & BP & $-20.03 * *$ & $-27.05^{* *}$ & $-40.46^{* *}$ & $-49.83 * *$ & $-28.50^{* *}$ & $-26.30 * *$ & 1.01 & $-3.81 * *$ & $-24.79 * *$ \\
\hline & SP & $18.92 * *$ & $14.19 * *$ & $-13.56^{* *}$ & -1.26 & $-9.29 * *$ & $23.46^{* *}$ & $-3.21 * *$ & $73.00 * *$ & $-13.90^{* * *}$ \\
\hline \multirow[t]{3}{*}{ L3 x T2 } & MP & $8.57 * *$ & $21.45^{* *}$ & $-22.156 * *$ & $-5.13 * *$ & $-3.97 * *$ & $-15.80 * *$ & $-5.95 * *$ & $-38.81 * *$ & 23.60 ** \\
\hline & BP & $3.10 * *$ & $17.06^{* *}$ & $-28.93 * *$ & $-10.34 * *$ & $-4.34 * *$ & $-17.02 * *$ & $-10.10 * *$ & $-39.01 * *$ & -1.49 \\
\hline & SP & $52.58 * *$ & $47.27 * *$ & $3.11 * *$ & $51.94 * *$ & $22.32 * *$ & $43.24 * *$ & $-5.53 * *$ & $10.46^{* *}$ & $-19.48^{* * *}$ \\
\hline \multirow[t]{3}{*}{ L3 x T3 } & MP & $-9.81 * *$ & 0.44 & $-18.505^{* *}$ & $-17.10 * *$ & $-13.42 * *$ & $-9.52 * *$ & $-8.05^{* *}$ & $14.34 * *$ & $-39.46^{* * *}$ \\
\hline & BP & $-10.91 * *$ & $-9.65 * *$ & $-26.78 * *$ & $-17.23 * *$ & $-18.08 * *$ & $-18.13 * *$ & $-8.89 * *$ & $2.83 * *$ & $-39.90 * *$ \\
\hline & SP & $35.14 * *$ & $32.02 * *$ & $6.17 * *$ & $40.28 * *$ & $3.93 * *$ & $37.23 * *$ & $-11.12 * *$ & $84.88 * *$ & $-50.28 * *$ \\
\hline L3 x T4 & MP & $3.70 * *$ & $12.16^{* *}$ & $6.575^{* *}$ & $17.84 * *$ & $-8.84 * *$ & $-1.31 * *$ & -0.39 & $22.30 * *$ & $-43.93 * *$ \\
\hline & BP & $-13.12 * *$ & $4.11^{* *}$ & $-9.98 * *$ & $-6.31 * *$ & $-18.50 * *$ & $-21.22 * *$ & $-2.51 * *$ & $-4.84 * *$ & $-49.07 * *$ \\
\hline & SP & 28.50 ** & $21.67 * *$ & $30.65^{* *}$ & $58.76^{* * *}$ & $3.40 * *$ & $31.97 * *$ & $-2.44 * *$ & $71.09 * *$ & $-49.07 * *$ \\
\hline L4 x T1 & MP & $-12.77 * *$ & $-3.65 * *$ & $-24.887 * *$ & $-27.22 * *$ & $-35.39 * *$ & $-17.67 * *$ & $-10.09 * *$ & $-10.77 * *$ & $-34.02 * *$ \\
\hline & BP & $-21.24 * *$ & $-18.36^{* *}$ & $-27.14 * *$ & $-36.73 * *$ & $-44.86^{* *}$ & $-31.58 * *$ & $-21.47 * *$ & $-30.49 * *$ & $-43.43 * *$ \\
\hline & SP & $17.20^{* *}$ & $27.99 * *$ & $-2.70 * *$ & $24.52 * *$ & $-28.37 * *$ & $3.17 * *$ & $-13.43 * *$ & $5.37 * *$ & $-35.25 * *$ \\
\hline L4 x T2 & MP & $-1.39 *$ & -0.49 & $-31.992 * *$ & $-32.04 * *$ & $2.48^{* *}$ & $-0.96^{*}$ & $-11.65 * *$ & $-18.87 * *$ & $-25.06^{* * *}$ \\
\hline & BP & $-6.28 * *$ & $-7.24 * *$ & $-35.50 * *$ & $-33.28 * *$ & $1.67 * *$ & $-7.25 * *$ & $-13.71 * *$ & $-25.46^{* *}$ & $-40.32 * *$ \\
\hline & SP & $24.82 * *$ & $16.78^{* * *}$ & $-13.87 * *$ & 0.67 & $32.09^{* * *}$ & $60.02 * *$ & $-4.95 * *$ & $34.84 * *$ & $-51.25^{* *}$ \\
\hline L4 x T3 & MP & $11.52 * *$ & $9.97 * *$ & $56.988 * *$ & $74.46^{* *}$ & $-66.75^{* *}$ & $-35.76^{* *}$ & $-34.84 * *$ & $9.74 * *$ & $-22.73 * *$ \\
\hline & BP & -0.16 & $-4.07 * *$ & $46.41 * *$ & $62.30 * *$ & $-68.88^{* *}$ & $-38.95 * *$ & $-38.57 * *$ & $6.85^{* *}$ & $-23.22 * *$ \\
\hline & SP & $51.35^{* *}$ & $40.08 * *$ & $95.66^{* *}$ & $174.17 * *$ & $-59.58 * *$ & $-8.10 * *$ & $-32.33 * *$ & $61.98 * *$ & $-36.46 * *$ \\
\hline L4 x T4 & MP & $-5.55^{* *}$ & $8.17 * *$ & $-2.947 * *$ & $4.38^{* *}$ & $27.14 * *$ & $3.76^{* *}$ & $5.93 * *$ & $52.87 * *$ & $-34.90 * *$ \\
\hline & BP & $-13.39 * *$ & $3.79 * *$ & $-15.15^{* *}$ & $-11.91 * *$ & $12.50^{* * *}$ & $-13.68 * *$ & $1.05^{*}$ & $26.85^{* * *}$ & $-40.83^{* * *}$ \\
\hline
\end{tabular}




\begin{tabular}{|c|c|c|c|c|c|c|c|c|c|c|}
\hline & SP & $3.93 * *$ & $13.04 * *$ & $13.36^{* *}$ & $28.04 * *$ & $46.16^{* *}$ & $29.97 * *$ & $11.25 * *$ & $92.30 * *$ & $-40.82 * *$ \\
\hline \multirow[t]{3}{*}{ L5 x T1 } & MP & $-17.17 * *$ & $-17.55^{* *}$ & $-1.936^{* *}$ & $-19.76 * *$ & $-5.76 * *$ & $4.79 * *$ & $-21.38^{* *}$ & $83.11 * *$ & $-24.21 * *$ \\
\hline & BP & $-24.71 * *$ & $-29.25 * *$ & $-6.42 * *$ & $-33.79 * *$ & $-20.01 * *$ & $-5.81 * *$ & $-31.33 * *$ & $58.18 * *$ & $-32.56 * *$ \\
\hline & SP & $12.04 * *$ & $10.74 * *$ & $17.49 * *$ & $30.29 * *$ & $5.29 * *$ & $17.70^{* *}$ & $-24.42 * *$ & $83.81 * *$ & $-22.88 * *$ \\
\hline \multirow[t]{3}{*}{ L5 x T2 } & MP & $-2.28 * *$ & -0.28 & $-15.955 * *$ & $-16.28 * *$ & $31.39 * *$ & $3.14 * *$ & $14.28 * *$ & $27.34 * *$ & $15.02 * *$ \\
\hline & BP & $-6.46 * *$ & $-5.71 * *$ & $-17.96^{* *}$ & $-22.63 * *$ & 29.51** & $-11.07 * *$ & $11.61^{* *}$ & $4.57 * *$ & $-11.23 * *$ \\
\hline & SP & $24.57 * *$ & $18.79 * *$ & $-1.63 *$ & $16.73 * *$ & $70.47 * *$ & $53.51 * *$ & $23.01 * *$ & $89.33 * *$ & $-20.69 * *$ \\
\hline \multirow[t]{3}{*}{ L5 x T3 } & MP & $-5.09 * *$ & $-5.05 * *$ & $2.642 * *$ & $-2.62 * *$ & $-9.28 * *$ & $3.78 * *$ & $-8.79 * *$ & $31.47 * *$ & -1.08 \\
\hline & BP & $-14.47 * *$ & $-16.08 * *$ & $1.99 * *$ & $-14.43 * *$ & $-15.62 * *$ & -0.31 & $-14.00^{* *}$ & $18.95 * *$ & $-4.62 * *$ \\
\hline & SP & $29.73 * *$ & $22.53 * *$ & $17.95^{* *}$ & $44.54 * *$ & $11.06^{* *}$ & $35.23 * *$ & $-5.33 * *$ & $70.88 * *$ & $-14.87 * *$ \\
\hline \multirow[t]{3}{*}{ L5 $\times$ T4 } & MP & $7.87 * *$ & $-12.43 * *$ & $-13.741 * *$ & $-24.69 * *$ & $-3.18 * *$ & $-5.64 * *$ & $12.11 * *$ & $19.28 * *$ & $-34.73 * *$ \\
\hline & BP & $-1.75 * *$ & $-17.18 * *$ & $-19.08 * *$ & $-32.92 * *$ & $-14.81^{* *}$ & $-15.09 * *$ & $6.94 * *$ & $10.94 * *$ & $-38.24 * *$ \\
\hline & SP & $19.66^{* *}$ & $-7.10 * *$ & $-7.60 * *$ & $-14.15 * *$ & $12.14 * *$ & $6.18 * *$ & 17.80 ** & $28.90 * *$ & $-38.16 * *$ \\
\hline \multirow[t]{3}{*}{ L6 x T1 } & MP & $-20.80 * *$ & $-23.86^{* *}$ & $-37.088 * *$ & $-52.08 * *$ & $16.37 * *$ & $12.58 * *$ & $18.92 * *$ & $92.97 * *$ & $-18.54 * *$ \\
\hline & BP & $-22.99 * *$ & $-24.73 * *$ & $-39.51 * *$ & $-53.40 * *$ & -0.55 & $5.90 * *$ & $4.50 * *$ & $91.15 * *$ & $-21.96 * *$ \\
\hline & SP & $21.38 * *$ & $17.93 * *$ & -17.70 ** & -2.93 & $28.74 * *$ & $19.95^{* *}$ & $13.56^{* *}$ & $64.73 * *$ & $-10.75^{* *}$ \\
\hline \multirow[t]{3}{*}{ L6 x T2 } & MP & 0.45 & $-1.72 * *$ & $-35.233 * *$ & $-36.71 * *$ & $-9.47 * *$ & $-1.34 * *$ & $-7.10 * *$ & $-6.16 * *$ & $17.13 * *$ \\
\hline & BP & $-7.33 * *$ & $-10.46^{* *}$ & $-39.09 * *$ & $-45.43 * *$ & $-10.03 * *$ & $-18.33 * *$ & $-8.64 * *$ & $-30.73 * *$ & $-14.34 * *$ \\
\hline & SP & $45.95^{* *}$ & $37.20 * *$ & $-17.08^{* *}$ & $13.65 * *$ & $16.46^{* *}$ & $40.99 * *$ & -0.71 & $25.30 * *$ & $-10.27 * *$ \\
\hline \multirow[t]{3}{*}{ L6 x T3 } & MP & $-16.08^{* *}$ & $-18.74 * *$ & $21.337 * *$ & $-1.59 *$ & $-54.84 * *$ & $-21.33^{* *}$ & $-21.76^{* *}$ & $-12.61 * *$ & $-37.21 * *$ \\
\hline & BP & $-17.63 * *$ & $-20.60 * *$ & $12.22 * *$ & $-10.89 * *$ & $-57.67 * *$ & $-27.86^{* * *}$ & $-25.74 * *$ & $-30.07 * *$ & $-43.79 * *$ \\
\hline & SP & $29.73 * *$ & $21.67 * *$ & $52.68 * *$ & $85.60 * *$ & $-45.20 * *$ & $-2.09 *$ & $-19.41^{* *}$ & 0.49 & $-41.07 * *$ \\
\hline \multirow[t]{3}{*}{ L6 x T4 } & MP & $-1.65^{* *}$ & $-1.82 *$ & $-30.741 * *$ & $-34.12 * *$ & $22.32 * *$ & $5.56 * *$ & $15.22 * *$ & $55.67 * *$ & $-11.92 * *$ \\
\hline & BP & $-19.61 * *$ & $-18.85^{* *}$ & $-39.92 * *$ & $-51.24 * *$ & $8.40 * *$ & -0.59 & $10.65 * *$ & $44.95 * *$ & $-13.96 * *$ \\
\hline & SP & $26.54 * *$ & $24.26^{* *}$ & $-18.31^{* *}$ & 1.55 & $40.33^{* *}$ & $12.44 * *$ & $20.11 * *$ & $45.02 * *$ & $-9.78 * *$ \\
\hline \multirow[t]{3}{*}{ L7 x T1 } & MP & $-29.97 * *$ & $-30.95 * *$ & $-24.108 * *$ & $-47.80 * *$ & $-35.48 * *$ & $1.65 * *$ & $2.22 * *$ & 1.51 & $7.11 * *$ \\
\hline & BP & $-33.07 * *$ & $-35.01 * *$ & $-28.30 * *$ & $-53.41 * *$ & $-41.91 * *$ & $-5.91 * *$ & $-11.27 * *$ & $-13.74 * *$ & $-8.48 * *$ \\
\hline & SP & $9.34 * *$ & 1.82 & $-9.89 * *$ & $-8.30 * *$ & $-33.40 * *$ & $10.18^{* *}$ & -0.90 & $4.31 *$ & $4.77 * *$ \\
\hline \multirow[t]{3}{*}{ L7 x T2 } & MP & $5.09 * *$ & $20.36^{* *}$ & $-3.149 * *$ & $16.76^{* * *}$ & $13.65^{* * *}$ & $-8.53 * *$ & $2.88 * *$ & $-21.63 * *$ & $-7.23 * *$ \\
\hline & BP & $-4.62 * *$ & $14.98 * *$ & $-6.43 * *$ & $15.39 * *$ & $7.78 * *$ & $-23.21 * *$ & -0.23 & $-34.64 * *$ & $-25.90 * *$ \\
\hline & SP & $55.77 * *$ & $59.06^{* *}$ & $12.14 * *$ & $78.25 * *$ & $37.81 * *$ & $32.47 * *$ & $11.44 * *$ & $18.30 * *$ & $-39.85 * *$ \\
\hline \multirow[t]{3}{*}{ L7 x T3 } & MP & $-10.01 * *$ & $-12.27 * *$ & $103.499 * *$ & 78.43** & $-78.40 * *$ & $-49.37 * *$ & $-44.24 * *$ & $-52.28 * *$ & $28.64 * *$ \\
\hline & BP & $-13.20 * *$ & $-14.63 * *$ & $100.09 * *$ & 70.81** & $-78.53 * *$ & $-52.83 * *$ & $-47.79 * *$ & $-56.05^{* *}$ & $27.32 * *$ \\
\hline & SP & $41.77 * *$ & $24.83 * *$ & $131.31 * *$ & $188.52 * *$ & $-75.39 * *$ & $-35.89 * *$ & $-41.58^{* *}$ & $-36.82 * *$ & $5.50 * *$ \\
\hline \multirow[t]{3}{*}{ L7 x T4 } & MP & $2.99 * *$ & $6.56^{* *}$ & $30.829 * *$ & $38.16^{* *}$ & $3.05 * *$ & -0.69 & $-2.76 * *$ & $-8.19 * *$ & $11.38 * *$ \\
\hline & BP & $-16.96^{* *}$ & $-8.18 * *$ & $23.96 * *$ & $13.80 * *$ & $-3.53 * *$ & $-7.98 * *$ & $-7.88 * *$ & $-16.13 * *$ & 0.89 \\
\hline & SP & $35.63 * *$ & $26.84 * *$ & $38.45 * *$ & $75.78 * *$ & $10.59 * *$ & $7.93 * *$ & $2.96 * *$ & 1.34 & $0.89 * *$ \\
\hline
\end{tabular}

$* *=$ Significance at $1 \%$ level; $*=$ Significance at $5 \%$ level

Standard heterosis for truss per plant was ranged from 131.31\% (Arka Saurabh $\times$ Alisa Craig) to $-18.31 \%$ (Arka Vikas $\times$ Pusa Ruby), the extent of heterosis over better parent was ranged from $-40.46 \%$ (Arka Ashish $\times$ Patharkutchi) to $100.09 \%$ (Arka Saurabh $\times$ Alisa Craig) and the magnitude of average heterosis ranged from $-37.09 \%$ (Arka Vikas $\times$ Patharkutchi) to $103.50 \%$ (Arka Saurabh $\times$ Alisa Craig). For fruits per plant the cross Arka Saurabh $\times$ Alisa Craig displayed highest values of average heterosis (78.43\%), Heterobeltiosis $(70.81 \%)$ and Standard heterosis (188.52\%). Similarly, for polar diameter highest values of Mid-parent heterosis $(12.58 \%)$ and better parent heterosis $(5.90 \%)$ in the cross Arka Vikas $\times$ Patharkutchi and standard parent heterosis $(60.02 \%)$ in the cross Arka Ahuti $\times$ Berika. Arka Alok $\times$ Patharkutchi hybrid displayed maximum values of heterosis over mid parent (32.24\%), better parent (14.12\%) and standard parent $(29.37 \%)$ for the trait equatorial diameter of the fruit. For fruit weight, range of average heterosis was from $78.40 \%$ (Arka Saurabh $\times$ Alisa Craig) to 
40.76\% (Arka Alok $\times$ Patharkutchi) and heterobeltiosis range was from $-78.53 \%$ (Arka Saurabh $\times$ Alisa Craig) to $29.51 \%$ (Arka Meghali $\times$ Berika). Similarly, the range of standard heterosis for fruit weight was from 75.39\% (Arka Saurabh $\times$ Alisa Craig) to $74.95 \%$ (Arka Alok $\times$ Berika). The extreme magnitude of average heterosis $(92.97 \%)$ and heterobeltiosis $(91.15 \%)$ for pericarp thickness was shown by cross Arka Vikas $\times$ Patharkutchi and standard heterosis $(92.72 \%)$ by Arka Alok $\times$ Alisa Craig. The range of magnitude of heterosis over mid parent ranged from 28.64\% (Arka Saurabh $\times$ Alisa Craig) to $-43.93 \%$ (Arka Ashish $\times$ Pusa Ruby), from $27.32 \%$ (Arka Saurabh $\times$ Alisa Craig) to $-49.07 \%$ (Arka Ashish $\times$ Pusa Ruby) over better parent and over standard parent from 5.50\% (Arka Saurabh $\times$ Alisa Craig) to $51.25 \%$ (Arka Ahuti $\times$ Berika) for the locule number. Low locule number indicates more dry matter, fruit firmness, suitable for long distance transportation and for processing. The discoveries in the present investigation were in conformation with the finding of Triveni et al., (2017) for flower per truss; Yogendra et al., (2013) for fruit per truss, (Saeed et al., 2014; Renuka and Sadashiva, 2016) for truss per plant, (Yashavantkumar, 2008; Sekhar et al., 2010) for fruits per plant, (Agarwal et al., 2014; Aisyah et al., 2016) for fruit weight, (Chauhan et al., 2014; Kumar et al., 2017) for polar diameter, (Yadav et al., 2013; Sherpa et al., 2014) for equatorial diameter, (Chattopadhyay and Paul, 2012; Renuka and Sadashiva, 2016) for pericarp thickness and (Mondal et al., 2009; Saeed et al., 2014; Mali and Patel 2014) for locule number. Considering all the heterosis manifested factors into an account the best cross identified was Arka Saurabh $\times$ Alisa Craig followed by Arka Alok $\times$ Berika, Arka Meghali $\times$ Berika and Arka Ahuti $\times$ Berika for the yield contributing traits which is due to the involvement of the parents having high GCA and crosses with good specific combining ability.

\section{References}

Agarwal, A., Arya D.N., Ranjan, R., and Ahmed, Z. 2014. Heterosis, combining ability and gene action for yield and quality traits in tomato (Solanum lycopersicum L.). Helix (Volume 1) by Various Artists on Spotify. 2, 511- 515 .

Aisyah, S. I., Wahyuni, S., Syukur, M., and Witono, J. R. 2016. The estimation of combining ability and heterosis effect for yield and yield components in tomato (Solanum lycopersicum L.) at lowland. Ekin Journal of Crop Breeding and Genetics. 2(1), 23-29.

Chattopadhyay, A., and Paul, A. 2012. Studies on heterosis for different fruit quality parameters in tomato. International Journal of Agriculture, Environment and Biotechnology. 5(4), 405-410.

Chattopadhyay, A., Dutta, S., Karmakar, K., Bhattacharya, I., and Hazra, P. 2007. "Technology for Vegetable Crop Production", Directorate of Research, BCKV, Kalyani, Nadia, West Bengal. Pp. 1-226.

Chauhan, V. B. S., Kumar, R., Behera, T. K. and Yadav, R. K. 2014. Studies on heterosis for yield and its attributing traits in tomato (Solanum lycopersicum L.). International Journal of Environment, Agriculture and Biotechnology. 7(1), 95100.

Duhan, D. V., Pratap, P. S., Rana, M. K., and Dahiya, M. S. 2005. Heterosis study for quality character in a line $\times$ tester set of tomato. Journal of Horticultural Sciences. 34(3), 371-375.

Fageria, M. S., Kholi, U. K., and Dhaka, R. S. 2001. Studies on heterobeltiosis for fruit and yield attributing traits in tomato (Lycopersicon esculentum Mill.). Journal of Horticultural Sciences. 30(2), 131-133.

Fernie, A. R., Tadmor, Y., and Zamir, D. 2006. Natural genetic variation for improving crop quality. Current Opinion in Plant Biotechnology. 9, 196-202.

Gami, R. A., Tank, C. J., Patel, S. S., Chauhan, R. 
M., and Patel, C. G. 2010. Genetic analysis for grain yield and quality in durum wheat (Triticum durum) under late sown condition. Green Farming. 1(6), 600-601.

Kempthorne, O. 1957. An Introduction to Genetic Statistics. John Wiley and Sons, Inc. New York. Pp. 208-223.

Kumar, M. S., Pal, A. K., Reddy, B. R., Anil, K., Singh, and Singh, A. K. 2017. Heterosis and Inbreeding Depression Studies for Yield and Yield Related Traits in Tomato (Solanum lycopersicum L.). International Journal of Current Microbiology and Applied Sciences. 6(11), 1240-1247.

Kumar, P., Neeraj, S., and Pradip, S. 2017. A Study on Heterosis in Tomato (Solanum lycopersicum L.) for Yield and its Component Traits. International Journal of Current Microbiology and Applied Sciences. 6(7), 1318-1325.

Mali, B., and Patel, A. I. 2014. Heterosis study in Tomato (Lycopersicon esculentum Mill.). Trends in Bioscience.7(4), 250-253.

Mondal, C., Sarkar, S., and Hazra, P. 2009. Line $\times$ Tester analysis of combining ability in tomato (Lycopersicon esculentum Mill.). Journal of Crop and Weed. 5 (1), 53-57.

Raj, T., Lal, B. M., and Pal, S. 2018. Estimation of heterosis for quality traits in tomato (Solanum lycopersicum L.). Journal of Pharmacognosy and Phytochemistry. 7(1), 2401-2404.

Raj, T., Madan L. B., Saheb, P., and Rajesh, K. D. 2017. Combining ability and gene action studies in some genetic stocks of tomato (Solanum lycopersicum L.). International Journal of Current Microbiology and Applied Sciences. 6(10), 138-144.

Renuka, D. M., and Sadashiva, A. T. 2016. Heterosis for growth, yield and quality traits in cherry tomato (Solanum lycopersicum var. cerasiforme). Plant Archives. 16(2), 654-658.

Saeed, A., Hasan, N., Shakeel, A., Saleem, M. F., Nazar H. K., Ziaf, K., Arif, R.M.K., and Saeed, N. 2014. Genetic analysis to find suitable parents for development of tomato hybrids. Life Science Journal. 11(12s), 30-35.

Savale, S. V., and Patel, A. I. 2017. Combining ability analysis for fruit yield and quality traits across environments in tomato (Solanum lycopersicum L.) International Journal of Chemical Studies. 5(5), 16111615.

Sekhar, L. L., Prakash, B. G., Salimath, P. M., Channayya, Hiremath, P., Sridevi, O., and Patil, A. A. 2010. Implications of heterosis and combining ability among productive Single cross hybrids in tomato. Electronic Journal of Plant Breeding. 1(4), 706-711.

Shankar, A., Reddy, R. V. S. K., Sujatha, M., and Pratap, M. 2013. Combining ability and gene action studies for yield and yield contributing traits in tomato (Solanum lycopersicum L.). Helix by Various Artists on Spotify. 1(6), 431-435.

Shende, V.D., Tania, S., Subhra, M., and Chattopadhyay, A. 2012. Breeding tomato (Solanum lycopersicum L.) for higher productivity and better processing qualities sabrao. Journal of Breeding and Genetics. 44(2), 302-321.

Sherpa, P., Seth, T., Shende, V.D., Pandiarana, N., Mukherjee, S., and Chattopadhyay, A. 2014. Heterosis, dominance estimate and genetic control of yield and post-harvest quality traits of tomato. Journal of Applied and Natural Science. 6(2), 625 632.

Tanksley, S. 1993. Linkage map of tomato (Lycopersicon esculentum) $(2 \mathrm{~N}=24)$. In: Genetic Maps: Locus Maps of Complex Genomes (Ed. O’Brien, J.), Cold Spring Harbor Laboratory Press, Cold Spring Harbor, New York. Pp. $39-60$.

Tesi, R., Grainfenberg, A., and Creatim, M. E. 1970. Heterosis and quality in $F_{1}$ hybrids of L. esculentum Mill, growth under glass. Riv. Ortoflorofruttic Ital. 54, 269292.

Thakur, A. K., Kholi, U. K., and Joshi, A. 2004. Evaluation of diallel progeny and heterosis for yield and yield components in tomato (Lycopersicon esculentum Mill.). Haryana Journal of Horticulture Science. 33(1/2), 106-108.

Triveni, D., Saidaiah, P., Reddy, K. R., and Pandravada, S. R. 2017. Studies on Heterosis for Yield and Yield 
Contributing Traits in Tomato (Solanum lycopersicum L.). International Journal of Pure and Applied Bioscience. 5(4), 1677 1685.

Triveni, D., Saidaiah, P., Reddy, K. R., and Pandravada, S. R. 2017. Studies on combining ability and gene action for growth and quality characters in tomato (Solanum lycopersicum L.). International Journal of Current Microbiology and Applied Sciences. 5(4), 1835-1840.

Wynne, J. C., Emery, D. A., and Rice, P. M. 1970. Combining ability estimates in Arachis hypogea L. II. Field performance hybrids. Crop Science. 10(6), 713-715.

Yadav, S. K., Singh, B. K., Baranwal, D. K., and Solankey, S. S. 2013. Genetic study of heterosis for yield and quality components in tomato (Solanum lycopersicum). African Journal of
Agricultural Research. 8(44), 5585-5591.

Yashavantakumar, K. H. 2008. Heterosis and combining ability for resistance against tospovirus in tomato (Solanum lycopersicon (Mill.) Wettsd.). M.Sc. (Agri.) Thesis, University of Agricultural Science, Dharwad.

Yogendra, K. N., and Gowda, P. H. R. 2013. Line $\times$ Tester analysis in tomato (Solanum lycopersicon L.) identification of superior parents for fruit quality and yield attributing traits. International Journal of Plant Breeding. 7(1), 50-54.

Zorzoli R., Guillermo R. P., Gustavo Ruben R., and Liliana A. P. 2007. Advances in Biotechnology: Tomato as a Plant Model System. Functional Plant Science and Biotechnology. 1 (1), 146-159.

\section{How to cite this article:}

Manjunath, K. V. and Subhamoy Sikder. 2020. Combining Ability, Gene Action and Heterotic Expression Studies for Yield Contributing Traits in Tomato. Int.J.Curr.Microbiol.App.Sci. 9(10): 2838-2850. doi: https://doi.org/10.20546/ijcmas.2020.910.342 